\title{
Spatio-temporal approach to moving window block kriging of satellite data v1.0
}

\author{
Jovan M. Tadić, Xuemei Qiu, Scot Miller, and Anna M. Michalak \\ Department of Global Ecology, Carnegie Institution for Science, Stanford, CA 94305, USA \\ Correspondence to: Jovan M. Tadić (jotadic@lycos.com)
}

Received: 15 July 2016 - Discussion started: 27 September 2016

Revised: 23 January 2017 - Accepted: 25 January 2017 - Published: 15 February 2017

\begin{abstract}
Numerous existing satellites observe physical or environmental properties of the Earth system. Many of these satellites provide global-scale observations, but these observations are often sparse and noisy. By contrast, contiguous, global maps are often most useful to the scientific community (i.e., Level 3 products). We develop a spatio-temporal moving window block kriging method to create contiguous maps from sparse and/or noisy satellite observations. This approach exhibits several advantages over existing methods: (1) it allows for flexibility in setting the spatial resolution of the Level 3 map, (2) it is applicable to observations with variable density, (3) it produces a rigorous uncertainty estimate, (4) it exploits both spatial and temporal correlations in the data, and (5) it facilitates estimation in real time. Moreover, this approach only requires the assumption that the observable quantity exhibits spatial and temporal correlations that are inferable from the data. We test this method by creating Level 3 products from satellite observations of $\mathrm{CO}_{2}\left(\mathrm{XCO}_{2}\right)$ from the Greenhouse Gases Observing Satellite (GOSAT), $\mathrm{CH}_{4}\left(\mathrm{XCH}_{4}\right)$ from the Infrared Atmospheric Sounding Interferometer (IASI) and solar-induced chlorophyll fluorescence (SIF) from the Global Ozone Monitoring Experiment2 (GOME-2). We evaluate and analyze the difference in performance of spatio-temporal vs. recently developed spatial kriging methods.
\end{abstract}

\section{Introduction}

Satellite observations of the Earth's surface and atmosphere provide a valuable window into the functioning of the Earth system. Satellites often provide global observations, but these observations are rarely uniform or contiguous in space- time. The observations can be non-contiguous due to satellite orbit geometries and periods, geophysical limitations (e.g., cloud cover), and temporary instrument malfunctions. Furthermore, satellites may provide a large quantity of data, but individual observations can have a large noise-to-signal ratio. It is often necessary to spatially interpolate the data in order to organize the data onto a regular grid, query the data at a particular location of interest, estimate data at unsampled times and/or locations, and/or map the underlying signal in a noisy dataset. These gridded, interpolated maps are commonly named "Level 3" data (e.g., NASA, 2014) and are often part of the standard suite of satellite data products.

$\mathrm{CO}_{2}$ column observations $\left(\mathrm{XCO}_{2}\right)$ from the Greenhouse Gases Observing Satellite (GOSAT), $\mathrm{CH}_{4}$ column observations $\left(\mathrm{XCH}_{4}\right)$ from the Infrared Atmospheric Sounding Interferometer (IASI) and solar-induced chlorophyll fluorescence (SIF) observations from the Global Ozone Monitoring Experiment-2 (GOME-2) provide prototypical examples of these challenges, and these three satellites are the primary application used throughout this work (see Sect. 3).

The most commonly used method for creating Level 3 maps from satellite data is binning. This approach involves taking the mean of all observations within a given grid cell or "bin" (see Kulawik et al., 2010, and Crévoisier et al., 2009a, for examples). The binning method, however, has a number of shortfalls that can lead to inconsistent or inaccurate results. First, different bins contain variable numbers of observations. As a result, some bins will be well constrained by the data, while others may be based upon sparse, noisy observations. Second, binning does not produce uncertainty estimates. Third, this method cannot extrapolate the unknown quantity to bins without any observations. 
A broad class of geostatistical methods known as kriging provides an alternative approach to mapping satellite observations. Kriging is a best linear unbiased estimator (for kriging, see Chiles and Delfiner, 2012), where covariance functions are used to represent correlations among data. As a result, kriging can account for a variable density of observations and can estimate uncertainties in the resulting maps. Various forms of kriging have recently been used to map satellite Earth observations, particularly for $\mathrm{XCO}_{2}$ (e.g., Hammerling et al., 2012a, b; Tadić et al., 2015; Zeng et al., 2013, 2016; Guo et al., 2013). Hammerling et al. (2012a, b) presented an approach to mapping Orbiting Carbon Observatory-2 (OCO-2) and GOSAT $\mathrm{XCO}_{2}$ observations, respectively, with non-stationary properties. In our previous study (Tadić et al., 2015) we extended that approach to create $\mathrm{XCO}_{2}$ maps that can have a different spatial resolution from the resolution or footprint of the original satellite observations. Our previous study and those of Hammerling et al. (2012a, b) accounted for spatial covariances among observations but did not include a temporal component. The present study extends this geostatistical framework from a purely spatial to a spatio-temporal domain.

Spatio-temporal approaches to interpolation can provide a number of advantages relative to purely spatial methods (e.g., Zeng et al., 2016; Guo et al., 2013). A purely spatial approach will usually aggregate observations into temporal blocks; observations within the same block effectively have the same time stamp whether or not those observations are actually synchronous (e.g., Tadić et al., 2015; Hammerling et al., 2012a, b). Any real temporal variability within a block becomes noise. A spatio-temporal approach, by contrast, treats time as an explicit dimension and models covariances among data as a function of time.

A handful of recent studies have considered temporal relationships when mapping satellite observations of $\mathrm{XCO}_{2}$. These studies have either used various forms of Kalman smoothing (e.g., Katzfuss and Cressie, 2011, 2012; Nguyen et al., 2014) or geostatistics (e.g., Guo et al., 2013; Zeng et al., 2013, 2016). The former group of studies leverages Kalman smoothing to improve the computational tractability of mapping dense or abundant datasets, like OCO-2 and the Atmospheric Infrared Sounder (AIRS). The latter group of studies, by contrast, has applied geostatistics to sparse datasets like those from the GOSAT satellite. A detailed review of spatial and spatio-temporal mapping methods has been published recently ( $\mathrm{Li}$ and Heap, 2014).

The goal of this study is to develop a geostatistical spatiotemporal mapping and upscaling method (applicable, but not limited, to satellite observations of $\mathrm{XCO}_{2}$ ) that exhibits several advances relative to previous methods. It can (1) fill in temporal gaps in the observations, (2) create maps at higher temporal resolutions than a purely spatial approach, (3) produce more accurate estimates when observations have variable spatio-temporal coverage, and (4) predict future values (i.e., extrapolate temporally). Among other improvements, we develop an efficient method for subsampling satellite observations and utilize the product-sum covariance model (e.g., De Iaco et al., 2001) that is easy to parameterize, which makes it applicable to both dense and sparse datasets. The entire work has been conducted in Matlab 2012a.

Section 2 of this study describes the presented model in detail; it describes an efficient subsampling procedure that can handle very large datasets and a covariance model that can estimate both spatial and temporal relationships in the data. We then incorporate these components into a spatio-temporal version of moving window block kriging. In Sects. 3 and 4, we subsequently apply this model to map GOSAT $\mathrm{XCO}_{2}$, IASI $\mathrm{XCH}_{4}$ and GOME-2 SIF at multiple time resolutions (including daily).

\section{Methods}

The spatio-temporal block kriging approach presented in this study proceeds in three steps for each model grid cell and estimation time. First, we subsample the observations within a predetermined spatio-temporal domain (Sect. 2.1). Next, we characterize the local spatio-temporal covariance structure (Sect. 2.2). Finally, we interpolate the satellite observations at the desired spatial resolution (Sect. 2.3).

\subsection{Subsampling of observations}

The ultimate goal of the proposed subsampling strategy is to reduce the number of observations in the spatio-temporal vicinity of an estimation location to a representative, computationally feasible subset of data. We use a subset of observations $(M)$ to estimate a local set of covariance parameters and use another subset $(N)$ to estimate the desired quantity and associated uncertainty. Note that, for the method presented here, $M$ and $N$ can refer to either the same subset of data or different subsets.

The total number of observations used for covariance parameter estimation $(M)$ is selected to be small enough to make this estimation computationally feasible but large enough to yield a sample representative of both local and regional variability. The optimal subset of $N$ observations used for mapping depends on the actually observed covariance structure, which is not known prior to the covariance parametrization step. In the example presented in Sect. 3, the optimal observational subset used in a mapping step for each grid cell comprised $N$ points having the highest covariance with the estimation location. In the example below, we set both $M$ and $N$ at 500; larger values of $M$ and $N$ did not have a substantial impact on the estimated parameters and mapped quantity, respectively.

We select a subset of observations $M$ for each estimation grid cell by assigning a relative selection probability to each observation based on that observation's spatial and temporal "separation distances" from the centroid of the grid cell. In 
the absence of a proper metric for distance in space-time, we model the spatial and temporal components of the overall selection probability separately.

The selection probability (and its components) is described by the following equation:

$P=P_{\mathrm{s}} \times P_{\mathrm{t}} \propto 1 /\left(A_{\mathrm{s}} h_{\mathrm{s}}\right)^{2} \times e^{-\left(A_{\mathrm{t}} h_{\mathrm{t}}\right)^{2}}$,

where $P_{\mathrm{S}}$ is the spatial component of the relative probability of a given observation being selected, $P_{\mathrm{t}}$ is the temporal component, $h_{\mathrm{s}}$ and $h_{\mathrm{t}}$ are distances between estimation location and observations, in space and time, respectively, and $A_{\mathrm{s}}$ and $A_{\mathrm{t}}$ are unit dependent, user defined weighting factors between separation distances in space vs. in time (how deep in space vs. time the sampling should occur). The unit dependent choice of $A_{\mathrm{s}}$ and $A_{\mathrm{t}}$ can be initially based on user expectations of the decorrelation distances in space vs. time and, if necessary, subsequently corrected, accounting for actually computed decorrelation lengths in space and time in an iterative fashion. In this way temporal and spatial sampling depths could even be locally optimized and become locationspecific. In the examples below (Sect. 3 ), $A_{\mathrm{s}}$ and $A_{\mathrm{t}}$ were set to $1 \mathrm{~km}^{-1}$ and $0.5 \mathrm{day}^{-1}$, respectively, based on the observed average decorrelation distances in space and time (see Fig. 1 and Sect. 4.1).

$h_{\mathrm{S}}$ is calculated as the great circle distance between the centroid $x_{j}$ of the estimation grid cell and the location $x_{i}$ of an observation:

$$
\begin{aligned}
h_{\mathrm{S}}\left(x_{i}, x_{j}\right) & =r \cos ^{-1}\left(\sin \varphi_{i} \sin \varphi_{j}\right. \\
& \left.+\cos \varphi_{i} \cos \varphi_{j} \cos \left(\lambda_{i}-\lambda_{j}\right)\right),
\end{aligned}
$$

where $\varphi_{i}$ and $\lambda_{i}$ are the latitude and longitude of location $x_{i}$ and $r$ is the radius of the Earth.

The temporal and spatial components of the probability function have different functional forms out of necessity. The measurements often come pre-aggregated in time slices corresponding to hours, days, or longer aggregation time periods, which multiplies the number of observations by the same time stamp. As a result, it is not possible to assign sampling probability along a temporal axis in a manner equivalent to the spatial approach; doing so would result in infinite probabilities assigned to all observations within the time slice of the actual estimation location $\left(P_{\mathrm{t}} \sim 1 / 0^{2}=\infty\right)$. The same holds for spatially co-located observations. However, since each observation comes with unique spatial coordinates (not pre-binned like in the temporal case), we select a simpler form of the spatial component of the sampling function. The defined form of $P$ (Eq. 1) ensures that pairs of observations close to the estimation location define the shape of the variogram at short separation distances (the variogram should reflect variability in the spatio-temporal vicinity of the estimation grid cell (see Sect. 2.2)). Different forms of $P$ can be used if directional anisotropy is expected or if more/fewer observations along a given direction are desired to better represent expected correlations.

Previous approaches required the user to choose spatial and temporal windows that determine which neighboring observations to use (see, for comparison, Alkhaled et al., 2008; Hammerling et al., 2012a, b). The approach proposed in this paper, by contrast, requires fewer subjective choices only the form of the sampling function and unit-dependent choice of normalizing coefficients $A_{\mathrm{s}}$ and $A_{\mathrm{t}}$. In addition, our approach is computationally feasible even for very large datasets.

\subsection{Characterization of spatio-temporal covariance}

Existing studies have used a number of models to estimate spatio-temporal covariances for a variety of applications. Models used include the metric model (Dimitrakopoulos and Luo, 1994), linear model (Rouhani and Hall, 1989), product model (De Cesare et al., 1996), non-separable model (Cressie and Huang, 1999), and generalized product-sum covariance model (De Iaco et al., 2001). The approach developed in this paper uses a generalized product-sum covariance model (De Iaco et al., 2001). This model affords a number of advantages relative to other covariance models: (1) a product sum covariance model outperformed other models in terms of prediction accuracy in a recent study using GOSAT satellite data (Guo et al., 2013), (2) it is relatively easy to implement (De Iaco et al., 2001), and (3) it is more flexible than a non-separable covariance model (De Cesare et al., 2001a).

The product-sum model, as it has been applied in the past, has one important area for improvement. The original procedure (De Iaco et al., 2001) assumed separate modeling of the spatial and temporal covariance (variograms) and their later unification into a spatio-temporal model in the final step. The procedure requires observations approximately in the same location at multiple different times. However, satellite observations are often not perfectly collocated in consequent measurement cycles over the same region. As a result, we would need to assume that each measurement cycle is perfectly colocated with previous/future cycles, or define an arbitrary tolerance, in order to apply the original approach. This assumption becomes more prone to error if the observations are very sparse, as is often the case with satellites.

Thus, in this study, we cater to specific properties of satellite data and alter the original procedure by estimating all covariance parameters simultaneously, thereby avoiding the aforementioned problem.

We broadly define the covariance as follows:

$C_{\mathrm{s}, \mathrm{t}}\left(h_{\mathrm{s}}, h_{\mathrm{t}}\right)=\operatorname{Cov}\left(Z\left(s_{+} h_{\mathrm{s}}, t_{+} h_{\mathrm{t}}\right), Z(s, t)\right)$.

The equation shows that covariance between two points $(Z)$ separated in space-time $(s, t)$ depends on their distance in space $\left(h_{\mathrm{s}}\right)$ and distance in time $\left(h_{\mathrm{t}}\right)$. The following class of valid product-sum covariance models was introduced in De Cesare et al. (2001b) and further developed in De Iaco et 

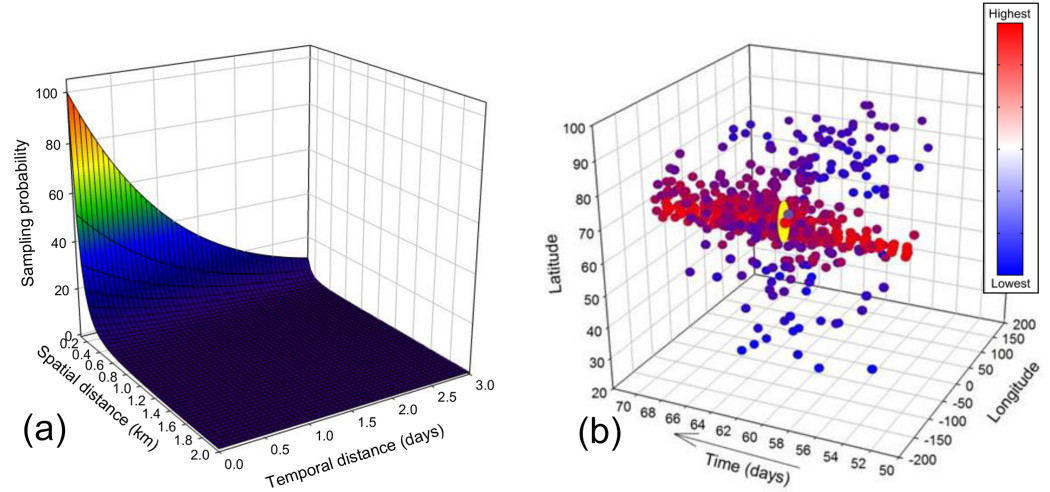

Figure 1. (a) Sampling probability as a decreasing function of spatial and temporal distance as used in this study. (b) The typical example of subsampled IASI Level $2 \mathrm{XCH}_{4}$ (altitude below $4 \mathrm{~km}$ ) data for a selected estimation location (yellow circle). The colors of the observations show semivariance between observation and estimation locations (blue: lowest; red: highest). Due to stronger temporal covariance, the relative decrease in the sampling probability along the temporal axis is smaller than with spatial distance.

al. (2001):

$C_{\mathrm{s}, \mathrm{t}}\left(h_{\mathrm{s}}, h_{\mathrm{t}}\right)=k_{1} C_{\mathrm{s}}\left(h_{\mathrm{s}}\right) C_{\mathrm{t}}\left(h_{\mathrm{t}}\right)+k_{2} C_{\mathrm{s}}\left(h_{\mathrm{s}}\right)+k_{3} C_{\mathrm{t}}\left(h_{\mathrm{t}}\right)$,

where $C_{\mathrm{t}}$ and $C_{\mathrm{s}}$ are valid temporal and spatial covariance models, respectively. De Iaco et al. (2001) proved that for positive definiteness it is sufficient that $k_{1}>0, k_{2} \geqslant 0$ and $k_{3} \geqslant 0$. It is interesting to note that from Eq. (4) it follows that spatio-temporal covariance models collapse down to purely spatial models in cases where temporal covariance does not exist. Thus, the spatial approach could be viewed as a special case of spatio-temporal modeling.

The model in Eq. (4) corresponds to the spatio-temporal variogram shown in Eq. (5). In the original procedure, De Iaco et al. (2001) estimated separate spatial $\left(h_{\mathrm{t}}=0\right)$ and temporal $\left(h_{\mathrm{s}}=0\right)$ variograms using the data. De Iaco et al. (2001) then combined these models to obtain the final spatio-temporal variogram model:

$$
\begin{aligned}
\gamma_{\mathrm{s}, \mathrm{t}}\left(h_{\mathrm{s}}, h_{\mathrm{t}}\right) & =\gamma_{\mathrm{s}, \mathrm{t}}\left(h_{\mathrm{s}}, 0\right)+\gamma_{\mathrm{s}, \mathrm{t}}\left(0, h_{\mathrm{t}}\right) \\
& -k \gamma_{\mathrm{s}, \mathrm{t}}\left(h_{\mathrm{s}}, 0\right) \gamma_{\mathrm{s}, \mathrm{t}}\left(0, h_{\mathrm{t}}\right),
\end{aligned}
$$

where $\gamma_{\mathrm{s}, \mathrm{t}}\left(h_{\mathrm{s}}, 0\right)$ and $\gamma_{\mathrm{s}, \mathrm{t}}\left(0, h_{\mathrm{t}}\right)$ are spatio-temporal variograms for $h_{\mathrm{t}}=0$ and $h_{\mathrm{s}}=0$, respectively (Fig. 2). Parameter $k$ is estimated from the data which make the model easily applicable:

$k=\frac{k_{\mathrm{s}} C_{\mathrm{s}}(0)+k_{\mathrm{t}} C_{\mathrm{t}}(0)-C_{\mathrm{s}, \mathrm{t}}(0,0)}{k_{\mathrm{s}} C_{\mathrm{s}}(0) k_{\mathrm{t}} C_{\mathrm{t}}(0)}$,

where $k_{\mathrm{s}} C_{\mathrm{s}}(0)$ and $k_{\mathrm{t}} C_{\mathrm{t}}(0)$ are spatial and temporal sills (variances) obtained in modeling of separate spatial and temporal variograms. The only condition $k$ has to fulfill in order to create an admissible covariance model is

$0<k \leqslant \frac{1}{\max \left\{\sigma_{\mathrm{s}}^{2}\left(\gamma_{\mathrm{s}, \mathrm{t}}\left(h_{\mathrm{s}}, 0\right)\right) ; \sigma_{\mathrm{t}}^{2}\left(\gamma_{\mathrm{s}, \mathrm{t}}\left(0, h_{\mathrm{t}}\right)\right)\right\}}$.

Due to the specifics of satellite data, we estimate both the covariance parameters and parameter $k$ simultaneously. This approach accounts for constraints that ensure a positive definiteness of the model (De Iaco et al., 2001). This simultaneous approach makes the model more applicable to sparse data and data with variable spatial coverage, as is often the case with satellite observations.

We use a Gaussian variogram function with a nugget effect to model temporal covariance in the example presented here (for an overview of variogram models, see Chiles and Delfiner, 2012). We use an exponential model for the spatial variogram. In both cases, we make this choice based upon visual inspection of local variograms at multiple estimation locations:

$$
\begin{aligned}
& \gamma_{\mathrm{t}}\left(h_{\mathrm{t}}\right) \text { (Gaussian) } \\
& =\left\{\begin{array}{r}
0, \text { for } h_{\mathrm{t}}=0, \\
\sigma_{t}^{2}\left(1-\exp \left(-\frac{h_{\mathrm{t}}^{2}}{l_{\mathrm{t}}^{2}}\right)+\sigma_{\text {nug }}^{2}, \text { for } h_{\mathrm{t}}>0,\right.
\end{array}\right. \\
& \gamma_{\mathrm{s}}\left(h_{\mathrm{s}}\right)(\text { exponential }) \\
& \quad=\left\{\begin{array}{r}
0, \text { for } h_{\mathrm{s}}=0, \\
\sigma_{\mathrm{s}}^{2}\left(1-\exp \left(-\frac{h_{\mathrm{s}}}{l_{\mathrm{s}}}\right)+\sigma_{\text {nug }}^{2}, \text { for } h_{\mathrm{s}}>0,\right.
\end{array}\right.
\end{aligned}
$$

where $\sigma^{2}$ and $l$ are the variance and correlation length of the quantity being mapped, and $\sigma_{\text {nug }}^{2}$ is the nugget variance, typically representative of measurement and retrieval errors in the case of satellite observations.

Unlike the original procedure in De Iaco et al. (2001), we model the variogram using only two steps. First, we calculate a raw spatio-temporal variogram based on the subsampled observations for each estimation grid cell:

$\gamma\left(h_{\mathrm{s}}, h_{\mathrm{t}}\right)=\frac{1}{2}\left[y\left(x_{i}\right)-y\left(x_{j}\right)\right]^{2}$,

where $\gamma$ is the raw spatio-temporal variogram value for a given pair of observations $y\left(x_{i}\right)$ and $y\left(x_{j}\right)$, and $h_{\mathrm{s}}$ and $h_{\mathrm{t}}$ are, respectively, the great circle distance and temporal distance 


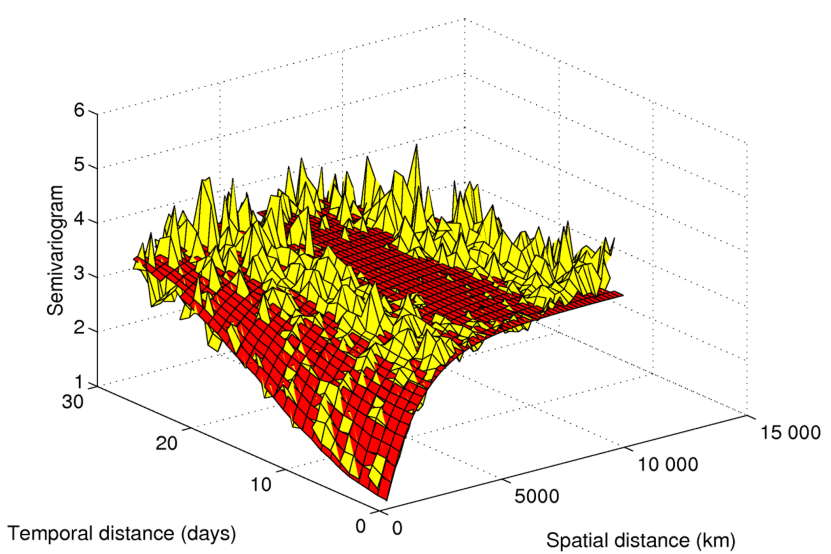

Figure 2. Illustration of the experimental and fitted theoretical spatio-temporal variogram for GOSAT $\mathrm{XCO}_{2}$ data.

between the spatio-temporal locations $\left(x_{i}\right.$ and $\left.x_{j}\right)$ of these observations.

Second, we fit the theoretical variogram defined in Eq. (5) to the raw variogram using non-linear least squares. We subsequently calculate the spatio-temporal covariance using the following equation:

$\left.C_{\mathrm{s}, \mathrm{t}}\left(h_{\mathrm{s}} h_{\mathrm{t}}\right)=C_{\mathrm{s}, \mathrm{t}}(0,0)-\gamma_{\mathrm{s}, \mathrm{t}}\left(h_{\mathrm{s}} h_{\mathrm{t}}\right)\right)$.

Validity on the sphere. Most covariance models were originally designed for Euclidean space, and their validity in other coordinate systems cannot be assumed per se. Huang et al. (2011) examined the validity of several theoretical covariance models in spherical coordinate systems. However, this evaluation has not been done for the spatio-temporal product-sum covariance model. Other studies that use a product-sum covariance model typically assume the validity of this covariance model on a sphere (e.g., Zeng et al., 2013, 2016). Results from Huang et al. (2011) explicitly validate the exponential covariance model on a sphere, as well as sums of the products of exponential covariance models and constants (provided that the constants are positive). The first term of the product-sum covariance model used in this study (Eq. 4) represents a Hadamard product (Million, 2007) of two positive definite matrices. According to the Schur product theorem, a Hadamard product of two positive definite matrices necessarily gives a positive definite matrix (Mathias, 1993). It therefore follows that a generalized product-sum model (Eq. 4) is valid on a sphere if its spatial component is valid on a sphere.

\subsection{Mapping using spatio-temporal moving window block kriging}

This section leverages the sampling function (Sect. 2.1) and the product-sum covariance model (Sect. 2.2) to implement a spatio-temporal version of moving window block kriging. A primary advantage of block kriging is its ability to estimate contiguous maps at any spatial resolution equal to or coarser than the spatial support (i.e., footprint size) of observations (refer to Sect. 1 and Tadić et al., 2015). Unlike the ordinary kriging method, the spatial support in block kriging corresponds to the average value within each chosen grid cell.

Moving window block kriging requires solving a set of linear equations to obtain a set of weights $(\lambda)$. These weights must be estimated for each prediction location using $\mathrm{N}$ associated observations:

$$
\left[\begin{array}{cc}
\mathbf{Q}+\mathbf{R} & \mathbf{1} \\
\mathbf{1}^{\mathrm{T}} & 0
\end{array}\right]\left[\begin{array}{c}
\lambda \\
-v
\end{array}\right]=\left[\begin{array}{c}
\boldsymbol{q}_{A} \\
1
\end{array}\right] \text {. }
$$

In this equation, $\mathbf{R}$ is a diagonal $N \times N$ nugget covariance matrix that describes measurement and retrieval errors, $\mathbf{Q}$ is an $N \times N$ covariance matrix among the $N$ observations with individual entries as defined in Eq. (11), 1 is an $N \times 1$ unity vector, $v$ is a Lagrange multiplier, and $\boldsymbol{q}_{A}$ is an $N \times 1$ vector of the spatio-temporal covariances between the $N$ observation locations and the estimation grid cell, defined as

$q_{A, i}=\frac{1}{n} \sum_{j=1}^{n} q\left(h_{\mathrm{s}_{i, j}}, h_{\mathrm{t}_{i, j}}\right)$,

where $q_{A, i}$ is the covariance between the grid cell and observation $i . q\left(h_{i, j}\right)$ is defined as $C_{\mathrm{s}, \mathrm{t}}$ in Eq. (11) based on the distances $h_{s_{i, j}}$ and $h_{\mathrm{t}_{i, j}}$ between observation $i$ and $n$ regularly spaced locations within the grid cell. In the context of satellite measurements, $n$ is the highest number of nonoverlapping footprints contained within a grid cell and was calculated based on the relative size of the satellite footprint compared to the size of the estimation grid cells. $n$ varies with latitude, as the size of grid cells decreases with the distance from the Equator. The system in Eq. (12) is solved for the weights $(\lambda)$ and the Lagrange multiplier $(v)$. We subsequently use these parameters to define the estimate $(\hat{z})$ and estimation uncertainty $\left(\sigma_{\hat{z}}^{2}\right)$ for the grid cell:

$\hat{z}=\lambda^{\mathrm{T}} \boldsymbol{y}$

$\sigma_{\hat{z}}^{2}=\sigma_{A A}-\lambda^{\mathrm{T}} \boldsymbol{q}_{A}+v$,

where $\boldsymbol{y}$ is the $N \times 1$ vector of subsampled observations, and $\sigma_{A A}$ is the variance of the observations at the resolution of the estimation grid cell, defined as

$\sigma_{A A}=\frac{1}{n^{2}} \sum_{j=1}^{n} \sum_{k=1}^{n} q\left(h_{j, k}\right)$.

In that equation, $q\left(h_{\mathrm{s}_{i, j}}, h_{\mathrm{t}_{i, j}}\right)$ is defined as $C_{\mathrm{s}, \mathrm{t}}$ in Eq. (11) based on the distances $h_{\mathrm{s}_{i, j}}$ and $h_{\mathrm{t}_{i, j}}$ between any combination of the $n$ regularly spaced locations within the grid cell defined previously.

\section{Example applications}

We select three case studies of satellite Level 2 data to demonstrate the properties of the method developed in this 

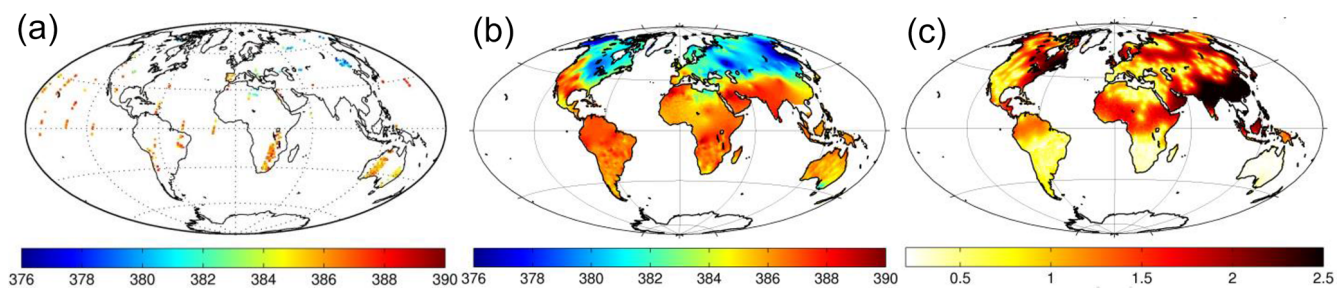

Figure 3. (a) GOSAT/ACOS v3.4 $\mathrm{XCO}_{2}$ retrievals (Level 2 data) (ppm) for 3 August 2009. (b) Contiguous global GOSAT/ACOS v3.4 maps (Level 3 data) (ppm) for the same day obtained using spatio-temporal moving window block kriging at $1 \times 1^{\circ}$ spatial resolution. (c) Associated uncertainties, given as $1 \sigma\left(\sigma_{\hat{z}}\right)(\mathrm{ppm})$.

paper: column-integrated dry air model fraction of $\mathrm{CO}_{2}$ $\left(\mathrm{XCO}_{2}\right)$ from the Japanese Greenhouse Gas Observing SATellite (GOSAT), $\mathrm{CH}_{4}\left(\mathrm{XCH}_{4}\right)$ from the Infrared Atmospheric Sounding Interferometer (IASI), and SIF from the Global Ozone Monitoring Experiment-2 (GOME-2). Level 2 datasets from GOSAT, IASI and GOME-2 have relatively different characteristics. For example, GOSAT observations are sparse, while IASI and GOME-2 are abundant. These diverse datasets are therefore ideal for testing the method developed here.

The method was demonstrated by producing two different sets of maps. First, it was applied at resolutions coarser than native $\left(1 \times 1^{\circ}, 2.5 \times 2^{\circ}\right.$, and $1 \times 1^{\circ}$ for GOSAT, IASI, and GOME-2, respectively) to demonstrate the block kriging capabilities of the method (Sect. 3). Second, it was applied at the native resolution of the satellites for cross-validation (method evaluation) purposes (Sect. 4).

\subsection{Total column $\mathrm{CO}_{2}\left(\mathrm{XCO}_{2}\right)$ observed by GOSAT}

The Japanese Greenhouse Gas Observing SATellite (GOSAT) (e.g., Kuze et al., 2009), the first satellite dedicated to global greenhouse gas monitoring, was launched in 2009. Basic information about the satellite, its orbit configuration, and the $\mathrm{CO}_{2}$ column observations are given in our previous study (Tadić et al., 2014). It flies in a polar, sun-synchronous orbit with a 3-day repeat cycle and an approximate 13:00 LT overpass time. GOSAT has a nadir footprint of about $10.5 \mathrm{~km}$ diameter at sea level (Kuze et al., 2009 ) and $2 \times 10^{3}$ observations per week. The $\mathrm{XCO}_{2}$ observations from GOSAT have large retrieval uncertainties (e.g., O'Dell et al., 2012) and exhibit large spatial and temporal gaps (e.g., Fig. 3a). Although these $\mathrm{XCO}_{2}$ observations are sparse and noisy, contiguous Level 3 maps are often desirable for environmental and ecological applications (Maksyutov et al., 2013; Liu et al., 2012). To this end, we generate global daily estimates for $\mathrm{XCO}_{2}$ (2-7 August 2009) to match the time frame used in Tadić et al. (2014).

We obtain bias-corrected and filtered GOSAT Level 2 observations using NASA's Atmospheric $\mathrm{CO}_{2}$ Observations from Space (ACOS) algorithm v3.4 release 3 (e.g., O’Dell et al., 2012; Crisp et al., 2012). In this study, we use spatiotemporal moving window block kriging to create a series of contiguous, in-filled global daily maps and associated uncertainties for 2-7 August 2009 (two repeat cycles) (Fig. 3a-c) at $1 \times 1^{\circ}$ resolution. We select the time period to match the time period from our previous study (Tadić et al., 2014). Unlike results from our previous study and other similar studies, which created estimates at 6-day or longer time periods (Hammerling et al., 2012a), we leverage the method developed here to produce maps at the daily scale.

\subsection{Total column $\mathrm{CH}_{4}\left(\mathrm{XCH}_{4}\right)$ observed by IASI}

The Infrared Atmospheric Sounding Interferometer (IASI) developed by the Centre National d'Etudes Spatiales (CNES) in collaboration with the European Organisation for the Exploitation of Meteorological Satellites (EUMETSAT) is a Fourier transform spectrometer based on a Michelson interferometer coupled to an integrated imaging system that measures infrared radiation emitted from the Earth. It is carried by MetOp-A, a sun-synchronous polar orbit satellite which flows at an altitude of $817 \mathrm{~km}$. Detailed information about the IASI instrument could be found elsewhere (Crévoisier et al., 2009a, b; Massart et al., 2014). IASI has an instantaneous field of view of $50 \times 50 \mathrm{~km}$, composed of four pixels each $12 \mathrm{~km}$ in radius, delivering $\sim 56 \times 10^{3} \mathrm{XCH}_{4}$ observations per week.

Methane Level 2 IASI $(0-4 \mathrm{~km})$ data were retrieved at the NOAA/NESDIS using the NUCAPS (NOAA Unique CrIS/ATMS Processing System) algorithm (Gambacorta, 2013; Xiong et al., 2013). For the ice-covered ocean the data for the lower troposphere $(0-4 \mathrm{~km})$ are unreliable due to insufficient thermal contrast between the surface and the atmosphere. Filtering parameters have been provided by X. Xiong (personal communication, 2014). The data are available at http://www.nsof.class.noaa.gov/. Using the new method, we created a series of contiguous global daily maps and associated uncertainties for the Northern Hemisphere, for 26 February-4 March 2013 (i.e., Fig. $4 a-c$ ) at $1^{\circ} \times 1^{\circ}$ resolution. We chose this time period to match the occurrence of the methane "anomaly" north of the coast of Scandinavia. 

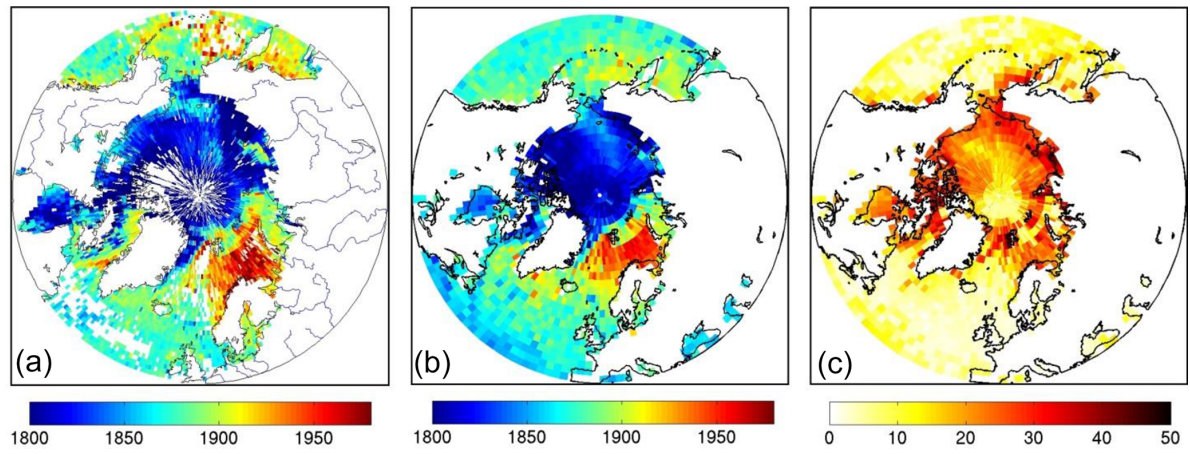

Figure 4. (a) IASI $\mathrm{XCH}_{4}(0-4 \mathrm{~km})$ retrievals (ppb) for 2 March 2013 (sea only). (b) Contiguous IASI maps for the Northern Hemisphere for the same day obtained using spatio-temporal moving window block kriging at $2.5 \times 2^{\circ}$ spatial resolution and (c) associated uncertainties, given as $1 \sigma\left(\sigma_{\hat{z}}\right)(\mathrm{ppb})$.

\subsection{Global land solar-induced fluorescence fields observed by GOME-2}

The GOME-2 (Global Ozone Monitoring Experiment-2) instrument on board METOP-A (e.g., Joiner et al., 2013) observes SIF. The GOME-2 spatial footprint (i.e., support) of the observations is $40 \mathrm{~km} \times 80 \mathrm{~km}$ (Joiner et al., 2013), and the volume of available data is approximately $2 \times 10^{5} \mathrm{SIF}$ observations per week.

Multiple recent studies have demonstrated the potential use of satellite observations of SIF for understanding the photosynthetic $\mathrm{CO}_{2}$ uptake at large scales (Joiner et al., 2011, 2012, 2013; Frankenberg et al., 2011, 2012, 2014; Guanter et al., 2012; Lee et al., 2013). Satellite SIF measurements can be used with land surface models to understand gross primary production (GPP) response to environmental stress (e.g., Lee et al., 2013) and to improve the representation of GPP. GOME-2 provides the highest spatial and temporal density of data, among all available datasets.

In the example presented here we use SIF GOME-2 v.14 data (Joiner et al., 2013) with the approach described in Sect. 2 to create contiguous maps of SIF at a single spatial resolution $\left(1^{\circ} \times 1^{\circ}\right)$ and daily temporal resolutions. Maps of SIF and associated uncertainties are created at daily temporal resolutions covering 5-14 May 2012, some of which are shown in Fig. 5a-c.

\section{Method evaluation: accuracy, precision and bias}

\subsection{Accuracy, precision and bias}

We use a leave-one-out cross-validation technique to assess the performance of spatio-temporal (ST) versus spatial moving window block kriging. We produce these estimates at the native resolution of GOSAT, IASI and GOME2 satellites/instruments, which allowed a direct comparison to measured values. For IASI and GOME-2, for each day in 26 February-4 March 2013, and 5-14 May 2012, respec- tively, $10 \%$ of available observational data were randomly selected for use in leave-one-out cross-validation, and their coordinates extracted. For $\mathrm{XCO}_{2}$, all GOSAT $\mathrm{XCO}_{2}$ observations for each day in 2-7 August 2009 were used. We assess the accuracy (the difference between estimates and withheld observations) of both methods using two common measures: (1) mean absolute error (MAE), and (2) root mean squared error (RMSE). We also use two more recently proposed measures ( $\mathrm{Li}$ and Heap, 2011; $\mathrm{Li}, 2016$ ) that remove the effect of unit/scale. The first is relative mean absolute error (RMAE), which is given as

$\mathrm{RMAE}=\frac{1}{n} \sum_{i=1}^{n}\left|\left(\hat{z}_{i}-y_{i}\right) / o_{i}\right| \times 100$,

and the second is relative root mean square error (RRMSE), as follows:

$\operatorname{RRMSE}=\left[\frac{1}{n} \sum_{i=1}^{n}\left(\left|y_{i}-\hat{z}_{i}\right| / y_{i}\right)^{2}\right]^{1 / 2} \times 100$,

where $n$ is the number of observations or samples, $o$ is the observed value, and $p$ is the predicted or estimated value.

We assess the performance of each method using two additional measures: (3) the accuracy of the uncertainty bounds (the degree to which the reported uncertainties capture the difference between estimates and withheld observations) and (4) bias (the mean difference between estimates and withheld observations).

We parameterize the temporal component of the spatiotemporal sampling function in such a way that observations located \pm 3 days from the actual date had a $10 \%$ probability of being sampled compared to observations from the actual day (see Fig. 1a). We compare the results to spatial kriging estimates obtained in two different ways, based on observations only from the actual day (1d) and based on observations from \pm 3 days from the actual day (7d). This latter case is analogous to the \pm 3 -day window that we use for the ST 


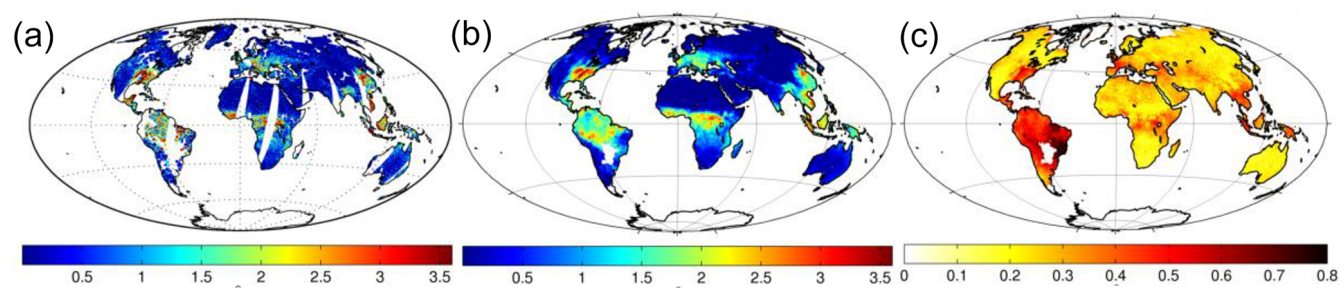

Figure 5. (a) GOME-2 SIF v14 retrievals (Level 2 data) $\left(\mathrm{mW} \mathrm{m}^{-2} \mathrm{sr}^{-1} \mathrm{~nm}^{-1}\right)$ for 5 May 2012, (b) contiguous global GOME-2/SIF v14 maps (Level 3 data) $\left(\mathrm{mW} \mathrm{m}^{-2} \mathrm{sr}^{-1} \mathrm{~nm}^{-1}\right)$ for the same day obtained using spatio-temporal moving window block kriging at $1 \times 1^{\circ}$ spatial resolution, and (c) associated uncertainties, given as $1 \sigma\left(\sigma_{\hat{z}}\right)\left(\mathrm{mW} \mathrm{m}^{-2} \mathrm{sr}^{-1} \mathrm{~nm}^{-1}\right)$.

approach. In this $7 \mathrm{~d}$ case, we obtain these spatial kriging results by assuming the entire observational dataset collected within the selected time period (actual day \pm 3 days) is perfectly temporally correlated. In other words, we use all observations as though they were collected at the same time. We then produce estimates at locations of observations collected within the selected time frame and compare the performance of the two methods. We repeat the procedure described in Sect. 2 for every observation selected for cross-validation, and we average the statistics, displayed in Table 1.

According to the results, the spatio-temporal approach performs better than the spatial (7d) approach in all three cases and in all performance measures (for example, the spatial (7d) MAE was 6-10\% larger). The comparison clearly shows that proper characterization of the temporal covariance between two points residing in different time periods (days), embedded in the spatio-temporal approach, improves kriging performance. In the IASI and GOME- 2 cases, the spatio-temporal method also performed better than the spatial one (1d). However, in the case of GOSAT data, the spatiotemporal approach slightly underperformed against the spatial (1d) approach, having a $12 \%$ higher MAE (please see Sect. 4.2 for discussion).

We observed that RMAE and RRMSE error measures should be used with caution in cases when observations can take real zero values, like in the GOME- 2 case. In such cases the division by close-to-zero values results in extremely high RMAE and RRMSE values, which overall limits the applicability of these error measures.

We evaluate the accuracy of the uncertainty bounds by examining how often those bounds encapsulate withheld observations. The percentage of observations that fall outside the uncertainty bounds in the spatio-temporal approach is comparable to that of the spatial method, confirming the accuracy of the estimated uncertainty bounds (for normally distributed data the percentage of observations that fall outside of the one, two, and three estimations standard deviation $\left(\sigma_{\hat{z}}\right)$ uncertainty bounds should be 32,5 , and $0.3 \%$, respectively). The fraction of observations that fall outside the uncertainty bound is generally lower than would be expected for normally distributed data, and our results may indicate nonnormal features in the data.

\subsection{When is spatio-temporal modeling recommended?}

An ST approach can afford advantages over purely spatial methods when temporal data correlations and data coverage are strong. Indeed, in many cases, the ST approach is more accurate than a purely spatial method (Table 1). This result is consistent with the existing literature, which uniformly reports that ST approaches are more accurate than spatial approaches (Zeng et al., 2013, 2016; Guo et al., 2013).

However, although considering information from days preceding and following the target estimation day should in principle always provide a further constraint on the estimate, this does not guarantee that an ST method will always outperform a spatial-only method in practice. The prime reasons for this are 2-fold. First, because computational limitations cap the number of observations that can be considered, considering observations across multiple days necessarily leads to a reduction in the spatial density of observations being considered. This first factor can be partially alleviated by carefully designing the selection probability function (Eq. 1). The second reason is that implementing an ST approach involves the estimation of a larger number of covariance parameters (Eqs. 4-9) relative to a spatial-only approach, which can introduce additional uncertainty. Indeed, we observe that the purely spatial approach performs better than the ST method in some cases (e.g., the GOSAT case).

Overall, an ST approach is likely to outperform a spatialonly approach when the data exhibit one (or more) of three characteristics. First, an ST approach is likely better when the data are sparse or unequally distributed. In these cases, an ST approach can intelligently leverage data in adjacent time periods to compensate for the sparsity of data in the time period of interest. Second, an ST approach works well for datasets with temporal gaps (e.g., due to cloud cover or instrument malfunction). An ST approach can fill these gaps, while a spatial-only approach cannot be used for temporal gap-filling. Third, an ST approach is well suited to datasets with regional biases that manifest in one time slice but that do not repeat in adjacent time slices. The differences between the performance of ST and S approaches obtained through cross-validation become most pronounced in processing datasets with measurement errors that are spatially 
Table 1. Cross-validation results of GOSAT $\mathrm{XCO}_{2}$, IASI $\mathrm{XCH}_{4}$ and GOME-2 SIF datasets using spatio-temporal and spatial methods, including mean absolute error (MAE), root mean squared error (RMSE), relative mean absolute error (RMAE), relative root mean square error (RRMSE), percent of observations lying outside of 1,2 , and 3 standard deviations $\left(\sigma_{\hat{z}}\right)$ of the mapping uncertainty, and mean difference. The MAE, RMSE and bias units for GOSAT, IASI and GOME-2 are ppm, ppb and $\mathrm{mW} \mathrm{m}^{-2} \mathrm{sr}^{-1} \mathrm{~nm}^{-1}$, respectively. RMAE and RRMSE are unitless, and, for the reasons explained in Sect. 4.1, given only for GOSAT and IASI. Shaded fields represent the best estimate in each category for every satellite.

\begin{tabular}{|c|c|c|c|c|c|c|c|c|c|c|}
\hline & & \multicolumn{3}{|c|}{ GOSAT $\mathrm{XCO}_{2}$} & \multicolumn{3}{|c|}{ IASI $\mathrm{XCH}_{4}$} & \multicolumn{3}{|c|}{ GOME-2 SIF } \\
\hline & & ST & $1 d$ & $7 d$ & ST & $1 d$ & $7 d$ & ST & $1 d$ & $7 \mathrm{~d}$ \\
\hline \multirow[t]{4}{*}{ Estimates } & $\begin{array}{l}\text { Mean absolute } \\
\text { error (MAE) }\end{array}$ & 0.83 & 0.74 & 0.89 & 19.19 & 20.23 & 21.04 & 0.52 & 0.54 & 0.54 \\
\hline & $\begin{array}{l}\text { Root mean squared } \\
\text { error (RMSE) }\end{array}$ & 1.12 & 0.98 & 1.21 & 25.25 & 27.10 & 27.77 & 0.68 & 0.69 & 0.69 \\
\hline & $\begin{array}{l}\text { Relative mean absolute } \\
\text { error (RMAE) }\end{array}$ & 0.22 & 0.19 & 0.23 & 1.04 & 1.09 & 1.14 & - & - & - \\
\hline & $\begin{array}{l}\text { Relative root mean } \\
\text { square error (RRMSE) }\end{array}$ & 0.29 & 0.25 & 0.31 & 1.37 & 1.46 & 1.50 & - & - & - \\
\hline \multirow[t]{3}{*}{ Uncertainties } & $\begin{array}{l}\% \text { observations falling } \\
\text { outside } 1 \sigma_{\hat{z}} \text { uncertainty }\end{array}$ & 9.13 & 15.03 & 10.70 & 11.02 & 9.06 & 13.84 & 14.60 & 12.14 & 24.80 \\
\hline & $\begin{array}{l}\% \text { observations falling } \\
\text { outside } 2 \sigma_{\hat{z}} \text { uncertainty }\end{array}$ & 1.12 & 3.01 & 1.39 & 0.48 & 0.51 & 0.86 & 1.20 & 0.64 & 4.33 \\
\hline & $\begin{array}{l}\% \text { observations falling } \\
\text { outside } 3 \sigma_{\hat{z}} \text { uncertainty }\end{array}$ & 0.067 & 0.52 & 0.13 & 0.04 & 0.046 & 0.022 & 0.11 & 0.05 & 0.83 \\
\hline Bias & Mean difference & -0.012 & 0.0066 & -0.034 & 0.28 & -0.14 & 0.58 & 0.016 & 0.0013 & 0.032 \\
\hline
\end{tabular}

but not temporally correlated. In these cases, an ST approach can use data from adjacent time periods to obtain an estimate, data that do not have the same regional, spatially correlated biases. Although the resulting estimate may appear inferior during cross-validation, this is because that estimate will not reproduce regional biases in data from the time slice of interest. A spatial-only approach, by contrast, will reproduce these regional biases because it does not use data from adjacent times when creating the estimate. As a result, a spatial-only approach will appear to perform better in crossvalidation, but the ST approach will more accurately reflect the true, underlying process.

\section{Conclusions}

In this study, we develop a method to create high spatiotemporal resolution maps from satellite data using spatiotemporal moving window block kriging based on the product-sum covariance model. The method relies on a limited number of assumptions: that the observed physical quantity is spatio-temporally auto-correlated, and that its nature can be inferred from the observations.

The method has several advantages over previously applied methods. Apart from the advances alluded to in Sect. 1, (1) it improves the covariance parameter estimation procedure because it does not model spatial and temporal covariance separately, (2) it allows for great flexibility in the choice of sampling function and (3) it provides estimates even for the time periods when measurements are not available. It can exploit correlations with both past and future periods of the observed time spot to provide the most accurate estimates.

We demonstrate the applicability of this method by creating Level 3 products from the GOSAT $\mathrm{XCO}_{2}$, IASI $\mathrm{CH}_{4}$ and GOME-2 SIF data. Sparse $\mathrm{XCO}_{2}$ observations from GOSAT and dense $\mathrm{XCH}_{4}$ and SIF observations from IASI and GOME-2 make a perfect test ground for the method. We show that the proposed method can even map $\mathrm{XCO}_{2}$ on daily timescales. The method generally yields more precise and accurate (and unbiased) estimates compared to the spatial method which used the same observations but assumed a perfect temporal correlation between the data. The factors which could affect the performance of the ST method are discussed in Sect. 4.2.

This approach could be used in the future to produce real-time estimates not only of $\mathrm{XCO}_{2}, \mathrm{XCH}_{4}$ or SIF, but of other environmental data observed by satellites which exhibit spatio-temporal autocorrelations. Especially important could be satellite datasets that have spatially, but not temporally, correlated errors. In such cases, sampling across several time periods could perhaps help isolate and remove them, which should be a subject of further studies.

The method could be applied in a standalone mode or as part of a broader satellite data processing package. Maps produced by the spatio-temporal approach could then be incorporated into physical and biogeochemical models of the Earth system. 


\section{Code availability}

The documented Matlab source code is available at the Researchgate website (https://www.researchgate.net/ publication/311595272_Spatio-temporal_approach_to_ moving_window_block_kriging_of_satellite_data_v10_ code; doi:10.13140/RG.2.2.21411.04643, Tadić et al., 2016). The code is made available under CC BY license terms (https://creativecommons.org/licenses/).

Competing interests. The authors declare that they have no conflict of interest.

Acknowledgements. This work was supported by the National Aeronautics and Space Administration (NASA) through grant nos. NNX12AB90G and NNX13AC48G, and the National Science Foundation (NSF) through grant no. 1342076. Satellite $\mathrm{CH}_{4}$ IASI v5 data are supplied by the NOAA National Environmental Satellite, Data, and Information Service (NESDIS): http://www.nsof.class.noaa.gov/. We would also like to thank Leonid Yurganov (JCET) and Nathaniel Lebedda (University of Maryland) for helpful information and discussions.

Edited by: L. Gross

Reviewed by: two anonymous referees

\section{References}

Alkhaled, A. A., Michalak, A. M., Olsen, S., Kawa, S. R., and Wang, J.-W.: A global evaluation of the regional spatial variability of column integrated $\mathrm{CO}_{2}$ distributions, J. Geophys. Res.Atmos., 113, D20303, doi:10.1029/2007JD009693, 2008.

Chiles, J.-P. and Delfiner, P.: Geostatistics, 2nd Edn., Wiley, New York, 2012.

Cressie, N. and Huang, H. C.: Classes of nonseperable, spatiotemporal stationary covariance functions, J. Am. Stat. Assoc., 94, 1-53, 1999.

Crevoisier, C., Nobileau, D., Fiore, A. M., Armante, R., Chédin, A., and Scott, N. A.: Tropospheric methane in the tropics - first year from IASI hyperspectral infrared observations, Atmos. Chem. Phys., 9, 6337-6350, doi:10.5194/acp-9-6337-2009, 2009a.

Crevoisier, C., Chédin, A., Matsueda, H., Machida, T., Armante, R., and Scott, N. A.: First year of upper tropospheric integrated content of $\mathrm{CO}_{2}$ from IASI hyperspectral infrared observations, Atmos. Chem. Phys., 9, 4797-4810, doi:10.5194/acp-9-4797-2009, 2009b.

Crisp, D., Fisher, B. M., O’Dell, C., Frankenberg, C., Basilio, R., Bösch, H., Brown, L. R., Castano, R., Connor, B., Deutscher, N. M., Eldering, A., Griffith, D., Gunson, M., Kuze, A., Mandrake, L., McDuffie, J., Messerschmidt, J., Miller, C. E., Morino, I., Natraj, V., Notholt, J., O’Brien, D. M., Oyafuso, F., Polonsky,
I., Robinson, J., Salawitch, R., Sherlock, V., Smyth, M., Suto, H., Taylor, T. E., Thompson, D. R., Wennberg, P. O., Wunch, D., and Yung, Y. L.: The ACOS $\mathrm{CO}_{2}$ retrieval algorithm - Part II: Global $\mathrm{XCO}_{2}$ data characterization, Atmos. Meas. Tech., 5, 687-707, doi:10.5194/amt-5-687-2012, 2012.

De Cesare, L., Myers, D. E., and Posa, D.: Spatio-temporal modelling of $\mathrm{SO}_{2}$ in Milan district, in: Geostatistics Wollongong, edited by: Baafi, E. Y. and Schofield, N. A., Kluwer Academic Publishing, Dordrecht, 1031-1042, 1996.

De Cesare, L., Myers, D. E., and Posa, D.: Product-sum covariance for space-time modeling: an environmental application, Environmetrics, 12, 11-23, 2001a.

De Cesare, L., Myers, D., and Posa, D.: Estimating and modeling space-time correlation structures, Stat. Probabil. Lett., 51, 9-14, 2001b.

De Iaco, S., Myers, D., and Posa, D.: Space-time analysis using a general product-sum model, Stat. Probabil. Lett., 52, 21-28, 2001.

Dimitrakopoulos, R. and Luo, X.: Spatiotemporal Modeling: Covariances and Ordinary Kriging Systems, Geostatistics for the Next Century, Kluwer Academic Publishers, Dordrecht, 88-93, 1994.

Frankenberg, C., Fisher, J. B., Worden, J., Badgley, G., Saatchi, S. S., Lee, J.-E., Toon, G. C., Butz, A., Jung, M., Kuze, A., and Yokota, T.: New global observations of the terrestrial carbon cycle from GOSAT: Patterns of plant fluorescence with gross primary productivity, Geophys. Res. Lett., 38, L17706, doi:10.1029/2011GL048738, 2011.

Frankenberg, C., O’Dell, C., Guanter, L., and McDuffie, J.: Remote sensing of near-infrared chlorophyll fluorescence from space in scattering atmospheres: implications for its retrieval and interferences with atmospheric $\mathrm{CO}_{2}$ retrievals, Atmos. Meas. Tech., 5, 2081-2094, doi:10.5194/amt-5-2081-2012, 2012.

Frankenberg, C., O’Dell, C., Berry, J., Guanter, L., Joiner, J., Köhler, P., Pollock, R., and Taylor, T. E.: Prospects for chlorophyll fluorescence remote sensing from the Orbiting Carbon Observatory-2, Remote Sens. Environ., 147, 1-12, doi:10.1016/j.rse.2014.02.007, 2014.

Gambacorta, A.: The NOAA Unique CrIS/ATMS Processing System (NUCAPS): Algorithm Theoretical Basis Documentation, NOAA Center for Weather and Climate Prediction (NCWCP), Version 1.0, available at: http://www.ospo.noaa.gov/Products/atmosphere/soundings/ nucaps/docs/NUCAPS_ATBD_20130821.pdf (last access: 10 February 2017), 2013.

Guanter, L., Frankenberg, C., Dudhia, A., Lewis, P. E., GomezDans, J., Kuze, A., Suto, H., and Grainger, R. G.: Retrieval and global assessment of terrestrial chlorophyll fluorescence from GOSAT space measurements, Remote Sens. Environ., 121, 236251, doi:10.1016/j.rse.2012.02.006, 2012.

Guo, L., Lei, L., and Zeng, Z.: Spatiotemporal correlation analysis of satellite-observed $\mathrm{CO}_{2}$ : Case studies in China and USA, Geoscience and Remote Sensing Symposium (IGARSS), 21-26 July 2013 IEEE International, Melbourne, VIC, 2013.

Hammerling, D. M., Michalak, A. M., and Kawa, S. R.: Mapping of $\mathrm{CO}_{2}$ at high spatiotemporal resolution using satellite observations: Global distributions from OCO-2, J. Geophys. Res., 117, D06306, doi:10.1029/2011JD017015, 2012a. 
Hammerling, D. M., Michalak, A. M., O'Dell, C., and Kawa, S. R.: Global $\mathrm{CO}_{2}$ distributions over land from the Greenhouse Gases Observing Satellite (GOSAT), Geophys. Res. Lett., 39, L08804, doi:10.1029/2012GL051203, 2012b.

Huang, C., Zhang, H., and Robeson, S. M.: On the validity of commonly used covariance and variogram functions on the sphere, Math. Geosci., 43, 721-733, doi:10.1007/s11004-011-9344-7, 2011.

Joiner, J., Yoshida, Y., Vasilkov, A. P., Yoshida, Y., Corp, L. A., and Middleton, E. M.: First observations of global and seasonal terrestrial chlorophyll fluorescence from space, Biogeosciences, 8, 637-651, doi:10.5194/bg-8-637-2011, 2011.

Joiner, J., Yoshida, Y., Vasilkov, A. P., Middleton, E. M., Campbell, P. K. E., Yoshida, Y., Kuze, A., and Corp, L. A.: Filling-in of near-infrared solar lines by terrestrial fluorescence and other geophysical effects: simulations and space-based observations from SCIAMACHY and GOSAT, Atmos. Meas. Tech., 5, 809-829, doi:10.5194/amt-5-809-2012, 2012.

Joiner, J., Guanter, L., Lindstrot, R., Voigt, M., Vasilkov, A. P., Middleton, E. M., Huemmrich, K. F., Yoshida, Y., and Frankenberg, C.: Global monitoring of terrestrial chlorophyll fluorescence from moderate-spectral-resolution near-infrared satellite measurements: methodology, simulations, and application to GOME2, Atmos. Meas. Tech., 6, 2803-2823, doi:10.5194/amt-6-28032013, 2013.

Katzfuss, M. and Cressie, N.: Spatio-temporal smoothing and EM estimation for massive remote-sensing data sets, Journal of Time Series Analysis, 32.4, 430-446, doi:10.1111/j.14679892.2011.00732.x, 2011.

Katzfuss, M. and Cressie, N.: Bayesian hierarchical spatio-temporal smoothing for very large datasets, Environmetrics, 23, 94-107, doi:10.1002/env.1147, 2012.

Kulawik, S. S., Jones, D. B. A., Nassar, R., Irion, F. W., Worden, J. R., Bowman, K. W., Machida, T., Matsueda, H., Sawa, Y., Biraud, S. C., Fischer, M. L., and Jacobson, A. R.: Characterization of Tropospheric Emission Spectrometer (TES) $\mathrm{CO}_{2}$ for carbon cycle science, Atmos. Chem. Phys., 10, 5601-5623, doi:10.5194/acp-10-5601-2010, 2010.

Kuze, A., Suto, H., Nakajima, M., and Hamazaki, T.: Thermal and near infrared sensor for carbon observation Fourier-transform spectrometer on the Greenhouse Gases 5 Observing Satellite for greenhouse gases monitoring, Appl. Optics, 48, 6716-6733, doi:10.1364/AO.48.006716, 2009.

Lee, J.-E., Frankenberg, C., van der Tol, C., Berry, J. A., Guanter, L., Boyce, C. K., Fisher, J. B., Morrow, E., Worden, J. R., Asefi, S., Badgley, G., and Saatchi, S.: Forest productivity and water stress in Amazonia: observations from GOSAT chlorophyll fluorescence, P. R. Soc. B, 280, 1762, doi:10.1098/rspb.2013.0171, 2013.

Li, J.: Assessing spatial predictive models in the environmental sciences: Accuracy measures, data variation and variance explained, Environ. Model. Softw., 80, 1-8, doi:10.1016/j.envsoft.2016.02.004, 2016.

Li, J. and Heap, A. D.: Spatial interpolation methods applied in the environmental sciences: a review, Environ. Model. Softw., 53, 173-189, doi:10.1016/j.envsoft.2013.12.008, 2014.

Li, J. and Heap, A.: A review of comparative studies of spatial interpolation methods in environmental sciences: performance and impact factors, Ecol. Inf., 6, 228-241, 2011.
Liu, J., Fung, I., Kalnay, E., Kang, J.-S., Olsen, E. T., and Chen, L.: Simultaneous assimilation of AIRS $\mathrm{XCO}_{2}$ and meteorological observations in a carbon climate model with an ensemble Kalman filter, J. Geophys. Res., 117, D05309, doi:10.1029/2011JD016642, 2012.

Maksyutov, S., Takagi, H., Valsala, V. K., Saito, M., Oda, T., Saeki, T., Belikov, D. A., Saito, R., Ito, A., Yoshida, Y., Morino, I., Uchino, O., Andres, R. J., and Yokota, T.: Regional $\mathrm{CO}_{2}$ flux estimates for 2009-2010 based on GOSAT and groundbased $\mathrm{CO}_{2}$ observations, Atmos. Chem. Phys., 13, 9351-9373, doi:10.5194/acp-13-9351-2013, 2013.

Massart, S., Agusti-Panareda, A., Aben, I., Butz, A., Chevallier, F., Crevoisier, C., Engelen, R., Frankenberg, C., and Hasekamp, O.: Assimilation of atmospheric methane products into the MACC-II system: from SCIAMACHY to TANSO and IASI, Atmos. Chem. Phys., 14, 6139-6158, doi:10.5194/acp-14-6139-2014, 2014.

Mathias, R.: Matrix completions, norms and Hadamard products, P. Am. Math. Soc., 117, 905-918, 1993.

Million, E.: The Hadamard Product, available at: http://buzzard.ups. edu/courses/2007spring/projects/million-paper.pdf (last access: 15 January 2015), 2007.

NASA Earth Science: available at: http:// science.nasa.gov/earth-science/earth-science-data/

data-processing-levels-for-eosdis-data-products/, last access: 23 July 2014.

Nguyen, H., Katzfuss, M., Cressie, N., and Braverman, A.: Spatio-Temporal Data Fusion for Very Large Remote Sensing Datasets, Technometrics, 56, 174-185, doi:10.1080/00401706.2013.831774, 2014.

O’Dell, C. W., Connor, B., Bösch, H., O’Brien, D., Frankenberg, C., Castano, R., Christi, M., Eldering, D., Fisher, B., Gunson, M., McDuffie, J., Miller, C. E., Natraj, V., Oyafuso, F., Polonsky, I., Smyth, M., Taylor, T., Toon, G. C., Wennberg, P. O., and Wunch, D.: The ACOS $\mathrm{CO}_{2}$ retrieval algorithm - Part 1: Description and validation against synthetic observations, Atmos. Meas. Tech., 5, 99-121, doi:10.5194/amt-5-99-2012, 2012.

Rouhani, S. and Hall, T. J.: Space-Time Kriging of Groundwater Data, in: Geostatistics, edited by: Armstrong, M., Kluwer Academic Publishers, Dordrecht, Vol. 2, 639-651, 1989.

Tadić, J. M., Loewenstein, M., Frankenberg, C., Butz, A., Roby, M., Iraci, L. T., Yates, E. L., Gore, W., and Kuze, A.: A comparison of in-situ aircraft measurements of carbon dioxide and methane to GOSAT data measured over Railroad Valley playa, Nevada, USA, IEEE T. Geosci. Remote Sens., 52, 7764-7774, doi:10.1109/TGRS.2014.2318201, 2014.

Tadić, J. M., Qiu, X., Yadav, V., and Michalak, A. M.: Mapping of satellite Earth observations using moving window block kriging, Geosci. Model Dev., 8, 3311-3319, doi:10.5194/gmd-8-33112015, 2015.

Tadić, J., Qiu, X., Miller, S., and Michalak, A. M.: Spatio-temporal approach to moving window block kriging of satellite data v1.0 code, doi:10.13140/RG.2.2.21411.04643, 2016.

Xiong, X., Barnet, C., Maddy, E. S., Gambacorta, A., King, T. S., and Wofsy, S. C.: Mid-upper tropospheric methane retrieval from IASI and its validation, Atmos. Meas. Tech., 6, 2255-2265, doi:10.5194/amt-6-2255-2013, 2013. 
Zeng, Z., LiPing, L. L., LiJie, G., Li, Z., and Bing, Z.: Incorporating temporal variability to improve geostatistical analysis of satellite-observed $\mathrm{CO}_{2}$ in China, Chinese Sci. Bull., 58, 19481954, 2013.

Zeng, Z., Lei, L., Strong, K., Jones, D. B. A., Guo, L., Liu, M., Deng, F., Deutscher, N. M., Dubey, M. K., Griffith, D. W. T., Hase, F., Henderson, B., Kivi, R., Lindenmaier, R., Morino, I., Notholt, J., Ohyama, H., Petri, C., Sussmann, R., Velazco, V. A., Wennberg, P. O., and Lin, H.: Global land mapping of satellite-observed $\mathrm{CO}_{2}$ total columns using spatiotemporal geostatistics, International Journal of Digital Earth, 131, doi:10.1080/17538947.2016.1156777, 2016. 